\title{
Characterization of Distribution by Conditional Expectation of Lower Record Values
}

\author{
M. I. Khan*, M. Faizan \\ Department of Statistics and Operations Research, Aligarh Muslim University, Aligarh, India \\ *Corresponding author: izhar.stats@gmail.com
}

Received November 31, 2013; Revised December 02, 2013; Accepted January 03, 2013

\begin{abstract}
It is widely known that the problem of characterizing a distribution an important problem which has recently attracted the attention of many researchers. Thus various characterizations have been established in many directions. In this paper, a general form of continuous probability distribution is characterized through conditional expectation of contrast of lower record statistics, conditioned on a non-adjacent record statistics and some of its deductions are also discussed.
\end{abstract}

Keywords: characterization, conditional expectation, continuous distributions, lower record values

Cite This Article: M. I. Khan, and M. Faizan, "Characterization of Distribution by Conditional Expectation of Lower Record Values." American Journal of Applied Mathematics and Statistics 2, no. 1 (2014): 7-9. doi: 10.12691/ajams-2-1-2.

\section{Introduction}

The record values were introduced by [1]. Suppose that $\left\{X_{i}\right\}_{i \geq 1}$ is a sequence of independent and identically distributed random variables with common distribution function $F(x)$ and common probability density function $f(x)$. Set $Y_{n}=\min (\max )\left\{X_{1}, X_{2}, \ldots, X_{n}\right\}$ for $n \geq 1$. We say $X_{j}$ is a lower (upper) record values of this sequence if $Y_{j}<(>) Y_{j-1}$ for $j>1$. By definition $X_{1}$ is a lower as well as upper record values and $L_{n}=\min \left\{j \mid j>L_{n-1}, Y_{j}<Y L_{n-1}, n \geq 2\right\} \quad$ with $\quad L_{1}=1$ denote the times of lower record values.

Record values are found in many situations of daily life as well as in many statistical applications. Often we are interested in observing new records, e.g. Olympic records. It is also useful in reliability theory, meteorology, hydrology, seismology, mining. For a more specific example, consider the situation of testing the breaking strength of wooden beams as described by [2].

For comprehensive accounts of the theory and applications of record values, we refer the readers to $[3,4,5,6]$.

\section{Objective}

Characterizing the distributions via their record statistics has a long history. For excellent review one may refer to [7-15] amongst others.

The aim of this paper is to characterize a general class of distributions via the contrast of the conditional expectation of function of lower record statistics, conditioned on non-adjacent lower record statistics.

\section{Method}

Let $X_{L(1)}, X_{L(2)}, \ldots, X_{L(r)}$ be the first $r$ lower record statistics from a population whose probability density function $p d f$ is $f(x)$ and the distribution function $(d f)$ is $F(x)$. Let $H(x)=-\log F(x)$ Then the $p d f$ of $X_{L(r)}, r=1,2, \ldots$ is

$$
f_{r}(x)=\frac{[H(x)]^{r-1} f(x)}{\Gamma(r)},-\infty<x<\infty
$$

and the joint $p d f$ of two lower records $X_{L(r)}$ and $X_{L(s)}$, $r<s, r, s=1,2, \ldots$ is

$$
\begin{aligned}
f_{r s}(x, y)= & \frac{1}{\Gamma(r) \Gamma(s-r)}[H(x)]^{r-1} \\
& \cdot[H(y)-H(x)]^{s-r-1} h^{*}(x) f(y)
\end{aligned}
$$

where $h^{*}(x)=-\frac{d H(x)}{d x}$.

Then the conditional $p d f$ of $X_{L(s)}$ given $X_{L(r)}=x, 1 \leq r<s$ is

$$
\begin{aligned}
& f\left(X_{L(s)} \mid X_{L(r)}=x\right) \\
= & \frac{1}{\Gamma(s-r)}[-\ln F(y)+\ln F(x)]^{s-r-1} \frac{f(y)}{F(x)}
\end{aligned}
$$

\section{Characterization Result}


Theorem: Let $X$ be an absolutely continuous random variable with the $d f F(x)$ and the $p d f f(x)$ on the support $(\alpha, \beta)$, where $\alpha$ and $\beta$ may be finite or infinite. Then for $1 \leq m<r<s \leq n$,

$$
\begin{gathered}
\sum_{i=r}^{m} c_{i} E\left[h\left(X_{L(l)}\right) \mid X_{L(s)}=x\right]=\frac{1}{a} \sum_{i=r}^{m} l c_{i}, \\
l=i-1, i
\end{gathered}
$$

if and only if

$$
F(x)=e^{-a h(x)}, x \in(\alpha, \beta), a>0
$$

where $c_{i}$ are real numbers $r \leq i \leq m$, satisfying $\sum_{i=r}^{m} c_{i}=0$, $c_{i} \neq 0$ for some $i$ and $h(x)$ is a non-increasing and differentiable function of $x$ such that $F(x)$ is a $d f$.

Proof: First we will prove (4.2) implies (4.1). We have from [14] $E\left[h\left(X_{L(i)}\right) \mid X_{L(s)}=x\right]=h(x)+\frac{i-s}{a} \quad$ for $F(x)=e^{-a h(x)}, x \in(\alpha, \beta), a>0$

Therefore,

$$
\begin{aligned}
& \sum_{i=r}^{m} c_{i} E\left[h\left(X_{L(i)}\right) \mid X_{L(s)}=x\right] \\
= & \sum_{i=r}^{m} c_{i}\left[h(x)+\frac{i-s}{a}\right] \\
= & \frac{1}{a} \sum_{i=r}^{m} i c_{i} \text { as } \sum_{i=r}^{m} c_{i}=0
\end{aligned}
$$

hence the 'if' part.

To prove the sufficiency part, we have

$$
\sum_{i=r}^{m} c_{i} E\left[h\left(X_{L(i)}\right) \mid X_{L(s)}=x\right]=\frac{1}{a} \sum_{i=r}^{m} i c_{i}
$$

or,

$$
\sum_{i=r}^{m} c_{i} \frac{a}{\Gamma(i-s)} \int_{\alpha}^{s} h(y)\left[\begin{array}{l}
-\ln F(y) \\
+\ln F(x)
\end{array}\right]^{i-s-1} \frac{f(y)}{F(x)} d y=\sum_{i=r}^{m} i c_{i}
$$

Integrating left hand side of (4.5) by parts, we get

$$
\begin{aligned}
& -\sum_{i=r}^{m} c_{i} \frac{a}{\Gamma(i-s)} \int_{\alpha}^{x} h^{\prime}(y)[-\ln F(y)+\ln F(x)]^{i-s-1} \frac{F(y)}{F(x)} d y \\
& +\sum_{i=r}^{m} c_{i} \frac{a}{\Gamma(i-s-1)} \int_{\alpha}^{x} h(y)\left[\begin{array}{l}
-\ln F(y) \\
+\ln F(x)
\end{array}\right]^{i-s-2} \frac{f(y)}{F(x)} d y \\
& =\sum_{i=r}^{m} i c_{i}
\end{aligned}
$$

That is,

$$
\begin{aligned}
& -\sum_{i=r}^{m} c_{i} \frac{a}{\Gamma(i-s)} \int_{\alpha}^{x} h^{\prime}(y)\left[\begin{array}{l}
-\ln F(y) \\
+\ln F(x)
\end{array}\right]^{i-s-1} \frac{F(y)}{F(x)} d y \\
& =\sum_{i=r}^{m} c_{i}(i-i+1)=0
\end{aligned}
$$

Now from (3.3), we have

$$
\frac{1}{\Gamma(i-s)} \int_{\alpha}^{x}[-\ln F(y)+\ln F(x)]^{i-s-1} \frac{f(y)}{F(x)} d y=1
$$

\begin{tabular}{|c|c|c|c|}
\hline Distribution & $F(x)$ & $a$ & $h(x)$ \\
\hline $\begin{array}{l}\text { Inverse } \\
\text { Weibull }\end{array}$ & $e^{-\theta x^{-p}} 0<x<\infty$ & $\theta$ & $x^{-p}$ \\
\hline $\begin{array}{l}\text { Power } \\
\text { function }\end{array}$ & $\left(\frac{x}{a}\right)^{p} \quad 0<x<a$ & $p$ & $-\log (x / a)$ \\
\hline Logistic & $\begin{array}{c}\left(1+e^{-x}\right)^{-1} \\
-\infty<x<\infty\end{array}$ & 1 & $\log \left(1+e^{-x}\right)$ \\
\hline Burr Type II & $\begin{array}{l}\left(1+e^{-x}\right)^{-k} \\
-\infty<x<\infty\end{array}$ & $k$ & $\log \left(1+e^{-x}\right)$ \\
\hline Burr Type III & $\begin{array}{l}\left(1+x^{-c}\right)^{-k} \\
0<x<\infty\end{array}$ & $k$ & $\log \left(1+x^{-c}\right)$ \\
\hline Burr Type IV & $\begin{array}{c}{\left[1+\left(\frac{c-x}{x}\right)^{1 / c}\right]^{-k}} \\
0<x<c\end{array}$ & $k$ & $\log \left[1+\left(\frac{c-x}{x}\right)^{1 / c}\right]$ \\
\hline Burr Type V & $\begin{array}{c}\left(1+c e^{-\tan x}\right)^{-k} \\
-\frac{\pi}{2}<x<\frac{\pi}{2}\end{array}$ & $k$ & $\log \left(1+c e^{-\tan x}\right)$ \\
\hline Burr Type VI & $\begin{array}{c}\left(1+c e^{-k \sinh x}\right)^{-k} \\
-\infty<x<\infty\end{array}$ & $k$ & $\log \left(1+c e^{-k \sinh x}\right)$ \\
\hline $\begin{array}{l}\text { Burr Type } \\
\text { VII }\end{array}$ & $\begin{array}{l}\left(\frac{1+\tanh x}{2}\right)^{k} \\
-\infty<x<\infty\end{array}$ & $k$ & $-\log \left(\frac{1+\tanh x}{2}\right)$ \\
\hline $\begin{array}{l}\text { Burr Type } \\
\text { VIII }\end{array}$ & $\begin{array}{l}\left(\frac{2}{\pi} \tan ^{-1} e^{x}\right)^{k} \\
-\infty<x<\infty\end{array}$ & $k$ & $-\log \left(\frac{2}{\pi} \tan ^{-1} e^{x}\right)$ \\
\hline Burr Type X & $\begin{array}{l}\left(1-e^{-x 2}\right)^{k} \\
0<x<\infty\end{array}$ & $k$ & $-\log \left(1-e^{-x 2}\right)$ \\
\hline Burr Type XI & $\begin{array}{c}\left(x-\frac{1}{2 \pi} \sin 2 \pi x\right)^{k} \\
0<x<1\end{array}$ & $k$ & $-\log \left(x-\frac{1}{2 \pi} \sin 2 \pi x\right)$ \\
\hline Gumbel & $\begin{array}{l}\exp \left[-e^{-x}\right] \\
-\infty<x<\infty\end{array}$ & 1 & $e^{-x}$ \\
\hline $\begin{array}{l}\text { Extreme } \\
\text { value II }\end{array}$ & $e^{-\left(\frac{\theta}{x}\right)^{p}} \quad 0<x<\infty$ & $\theta^{p}$ & $x^{-p}$ \\
\hline
\end{tabular}

Therefore,

$$
\sum_{i=r}^{m} c_{i} \frac{1}{\Gamma(i-s)} \int_{\alpha}^{x}\left[\begin{array}{l}
-\ln F(y) \\
+\ln F(x)
\end{array}\right]^{i-s-1} \frac{f(y)}{F(x)} d y=0
$$

Comparing (4.7) and (4.8), we get

$$
-a h^{\prime}(y) F(y)=f(y)
$$

implying

$$
F(y)=e^{-a h(y)}, a>0
$$

and hence the Theorem.

Remark: Putting $c_{m}=1$ and $c_{r}=-1$ in Theorem, we get the characterizing result as obtained by [14].

Table 4.1. Examples based on the distribution function $F(x)=e^{-a h(x)}, \quad a>0$ 


\section{Discussion}

The purpose of this paper was to characterize a general classs of probability distribution through the conditional expectation based on lower record statistics conditioned on non-adjacent lower record statistics using the contrast technique. We hope that findings of this paper will useful for the researcher in various fields. Further advancement of research in distribution theory, lower record theory and their application.

\section{References}

[1] K.N. Chandler (1952). The distribution and frequency of record values. Journal of Royal Society. B14, 220-228.

[2] Glick, N. (1978). Breaking record and breaking boards. American Mathematical Monthly, 85, 2-26.

[3] Ahsanullah, M. (1995). Record Statistics. Nova Science Publishers, New York.

[4] Arnold, B. C., Balakrishnan, N. and Nagaraja, H. N. (1998). Record . Johan Wiley and Sons, New York.

[5] Ahsanullah, M. (2004). Record Values-Theory and Applications.University Press of America, New York.

[6] Ahsanullah, M. and Raqab, M. Z. (2006). Bounds and Characterizations of Record Statistics. Nova Science Publishers, Hauppauge, New York.
[7] Malinowska, I. and Szynal, D. (2008). On characterization of certain dis-tributions of $k$ th lower (upper) record values. Applied Mathematics Computation, 202, 338-347.

[8] Shawky, A. I. and Bakoban, R. A. (2008). Characterization from exponentiated gamma distributions based on record values. Journal of Statistical Theory and Applications, 7, 263-277.

[9] Shawky, A. I. and Bakoban, R. A. (2009). Conditional expectation of certain distributions of record values. International Journal of Mathematical Analysis, 3 (17), 829-838.

[10] Shawky, A. I. and Abu-Zinadah, H. H. (2006). General recurrence relations and characterizations of certain distributions based on record values. Journal of Approximation Theory and Applications, 2, 149-159.

[11] Shawky, A. I. and Abu-Zinadah, H. H. (2008a). Characterization of the exponentiated Pareto distribution based on record values. Applied Mathematical Sciences, 2, 1283-1290.

[12] Shawky, A. I. and Abu-Zinadah, H. H. (2008b). General recurrence relations and characterizations of certain distributions based on record statistics. Journal of Statistical Theory and Applications, 7, 93-117.

[13] Wu, J.W. and Lee, W. C. (2001): On characterizations of generalized extreme values, power function, generalized Pareto and classical Pareto distributions by conditional expectation of record values. Statistical Papers, 42, 225-242.

[14] Faizan, M. and Khan, M. I.(2011). A characterization of continuous distributions through lower record statistics. ProabStat Forum, 4, 39-43.

[15] Nadarajah, S., Teimouri, M. and Shih, S.H. (2012). Characterizations of the Weibull and Uniform distributions using record values. Brazilian Journal of Probability and Statistics. (To appear). 